\section{Hämolytisch-urämisches Syndrom: Langzeitfolgen über Jahre beobachten}

Langzeitfolgen des hämolytisch-urämischen Syndroms (HUS) noch nach Jahren, neurologische Symptomatik bislang unterschätzt, lange Nachbeobachtung erforderlich - diese Erkenntnisse lieferte die Auswertung von Daten, die während der EHEC-Epidemie 2011 gewonnen worden waren. Auslöser des Ausbruchs war der bis dahin nur sporadisch aufgetretene Erreger EHEC O104:H4. 3.816 Menschen hatte der besonders aggressive, Shigatoxin-produzierende Keim infiziert, 845 Patienten entwickelten das Vollbild eines HUS - davon 90 Kinder. „Der weltweit größte Ausbruch bei Kindern überhaupt“, wie PD Dr. Jun Oh, Hamburg, berichtete.

Betroffen waren deutlich ältere Kinder mit durchschnittlich elf Jahren als in historischen Fällen (zwei bis vier Jahre). $96 \%$ entwickelten Diarrhö, die nur bei $70 \%$ blutig war. „Das Fehlen blutiger Durchfälle schließt ein HUS also nicht aus", so Oh. Hinsichtlich anderer Charakteristika zeigten sich keine wesentlichen Unterschiede: $70 \%$ der Kinder wurden dialysepflichtig im Median für elf Tage; schwere neurologische Symptome wie Krampfanfälle, Koma oder visuelle Probleme traten bei $23 \%$ auf. Therapeutisch kam die Plasmapherese bei $20 \%$ vor allem wegen neurologischer Komplikationen zum Einsatz, $14 \%$ wurden mit Eculizumab behandelt. Die Daten zur Behandlung mit dem Antikörper, der an Protein C5 des Komplementsystems bindet, stehen noch aus. Ein Kind musste nierentransplantiert werden, bei $4,4 \%$, die eine Niereninsuffizienz entwickelten, besserte sich die Nierenfunktion.

„Was aus diesen Patienten in den kommenden fünf bis zehn Jahren wird, das wird zu verfolgen sein“, sagte Oh vor dem Hintergrund aktueller Daten, die zeigen, dass $30 \%$ der Patienten fünf Jahre nach HUS noch unter Langzeitfolgen litten - etwa Hypertonus, eingeschränkte Nierenfunktion, Proteinurie oder neurologische Störungen. Auffällig dabei: Patienten, die bis zum dritten Jahr nach HUS symptomfrei waren, entwi- ckelten plötzlich zwei Jahre später erstmals Symptome, vor allem Proteinurie. Hinsichtlich neurologischer Symptome zeigte die jüngste Epidemie noch nach sechs Monaten pathologische EEGs bei $19 \%$, bei $48 \%$ Antriebsschwäche, Müdigkeit, Konzentrationsstörungen und Angstzustände - Symptome also, die jetzt betroffene ältere Kinder wesentlich besser beschreiben können als bislang betroffene jüngere. Oh: „Das zeigt, dass wir neurologische Symptome möglicherweise unterschätzt haben und dass wir unsere Patienten lange nachbeobachten müssen.“

Michael Koczorek

Oh J. Klinische Verläufe der EHEC-O104 H4-Infektion bei Kindern

\title{
Zweite MMR-Impfung: mehr Kinder früher vor Masern schützen
}

Die Ständige Impfkommission empfiehlt seit 2001 eine zweite Dosis mit MasernMumps-Röteln-Impfstoff im zweiten Lebensjahr. Bei diesem Konzept der zweiten Impfung gehe es nicht um einen Booster-Effekt, wie Prof. Dr. Ulrich Heininger, Basel, erklärte: „Es geht darum, primären Impfversagern eine zweite Chance zu geben." Denn etwa 5\% der Kinder sind nach der ersten Impfung nicht vor Masern geschützt, nach der zweiten Impfung sinkt die Rate auf 0,1 \%. Der Unterschied in den Schutzraten von $95 \%$ versus $99,9 \%$ erscheine auf den ersten Blick nicht hoch, aber, so Heininger:
„Nach der ersten Dosis ist jedes 20. Kind ungeschützt, nach der zweiten Dosis nur noch jedes 1.000. Kind.“ Epidemiologisch sei das ein großer Unterschied. Ein weiterer Vorteil der zweiten Dosis besteht in einer besseren Qualität der Immunantwort. Die Empfehlung, die zweite Impfung schon früh nach der ersten zu verabreichen, geschieht vor dem Hintergrund der hohen Maserninzidenz in den ersten beiden Lebensjahren.

Michael Koczorek

Heininger U. „Stiko-Empfehlungen im europäischen Vergleich"

\section{Gute Prognose bei Bauchwanddefekten}

Wie sind die Langzeitergebnisse nach der Therapie neonataler Krankheiten? Wie nachhaltig erfolgreich waren die Interventionen etwa nach angeborenen Bauchwanddefekten? Auch diesen Fragen ging die DGKJ gemeinsam mit der Gesellschaft für Neonatologie und Pädiatrische Intensivmedizin (GNPI) und der Deutschen Gesellschaft für Kinderchirurgie (DGKCH) nach. Dabei zeigte sich, dass dank enger Zusammenarbeit von Neonatologie sowie Kin- der- und Neugeborenenchirurgie komplexe Fehlbildungen inzwischen erfolgreich therapiert und die Langzeitprognosen der kleinen Patienten verbessert werden konnten. So haben über $90 \%$ der Patienten mit den häufigsten Bauchwanddefekten bei Neugeborenen - Omphalozele und Gastroschisis - einen komplikationsarmen Verlauf, so Prof. Dr. Konrad Reinshagen, Klinik für Kinderchirurgie am Altonaer Kinderkrankenhaus und am Universitätsklinikum
Hamburg-Eppendorf: „Bei neuropsychologischen Untersuchungen wurden zudem keine signifikanten psychomotorische Entwicklungsverzögerungen festgestellt.“ Den Eltern kann laut Reinshagen somit eine normale Lebenserwartung und eine normale Entwicklung der Psychomotorik prognostiziert werden. „Das ist natürlich bei der pränatalen Beratung der Eltern immens bedeutsam."

Birgit Frohn

Reinshagen K. Langzeitergebnisse nach kongenitalen Bauchwanddefekten 